\title{
A Retrospective Analysis of Serous Effusions Based on the Newly Proposed International System for Reporting Serous Fluid Cytopathology: A Report of 3633 Cases in an Oncological Center
}

\author{
Yan-li Zhu \\ Peking University Cancer Hospital \\ Wen-hao Ren \\ Peking University Cancer Hospital \\ Qian Wang \\ Peking University Cancer Hospital \\ Hai-zhu Jin \\ Peking University Cancer Hospital \\ Yi-yi Guo \\ Peking University Cancer Hospital \\ Dong-mei Lin ( $\sim 13621058958 @ 139 . c o m$ ) \\ Peking University Cancer Hospital
}

\section{Research Article}

Keywords: cytology, effusion, The International System for Reporting Serous Fluid, risk of malignancy, liquid-based cytology

Posted Date: January 12th, 2022

DOI: https://doi.org/10.21203/rs.3.rs-1234200/v1

License: (c) (1) This work is licensed under a Creative Commons Attribution 4.0 International License. Read Full License 


\section{Abstract}

Background: The International System for Reporting Serous Fluid Cytopathology (TIS) was recently proposed. We retrospectively applied TIS recommendations for reporting the cytological diagnosis of serous effusions and reported our experience.

Methods: All the serous effusions from January 2018 to September 2021 were retrieved from the database. Recategorization was performed using the TIS classification, the risk of malignancy (ROM) was calculated for each TIS category, and the performance evaluation was carried out among different samples (pleural, peritoneal and pericardial effusions) and preparation methods (conventional smears, liquid-based preparations and cellblocks).

Results: A total of 3633 cases were studied, 17 (0.5\%) were diagnosed as ND, 1100 (30.3\%) as NFM, 101 (2.8\%) as AUS, 677 (18.6\%) as SFM, and $1738(47.8 \%)$ as MAL. The ROM for the categories were found to be $38.5 \%, 28.6 \%, 52.1 \%, 99.4 \%$ and $100 \%$, respectively. The ROM for SFM was significantly higher than that for AUS $(\mathrm{P}<0.001)$, while the difference between the ROMs for the IVa and IVb was insignificant. The sensitivity, negative predictive value(NPV) and diagnostic accuracy of liquid-based preparations were all superior to conventional smears and cell-blocks in detecting abnormalities. Using three preparation methods simultaneously had the highest sensitivity, NPV and diagnostic accuracy.

Conclusion: Serous effusion cytology has a high specificity and positive predictive value(PPV), and the TIS is a user-friendly reporting system. Liquidbased preparations could improve the sensitivity of diagnosis, and it is best to use three different preparation methods simultaneously for serous effusion cytologic examination.

\section{Background}

Effusions can produce inside serous cavities in neoplastic and non-neoplastic lesions. Serous effusion cytology is a common clinical examination method to distinguish benign and malignant serous effusions for its advantages of minimally invasive, easily available, and effectively cost[1-3]. In view of the importance of cytology in the evaluation of effusion specimens, its role in patient management has become more and more important, an international group of cytopathology experts published "The International System for Reporting Serous Fluid Cytopathology" (TIS) to standardize the reporting terminology and criteria to establish diagnostic categories with high diagnostic value[4, 5].

The 5 proposed diagnostic categories are nondiagnostic (I, ND), negative for malignancy (II, NFM), atypia of undetermined significance (III, AUS), suspicious for malignancy (IV, SFM), and malignant (V, MAL). TIS has defined the AUS category as specimens that lack quantitative or qualitative cytologic features to be confidently diagnosed as either benign or malignant and that exhibit sufficiently clear morphologic features to exclude the possibility of classifying them as ND, and the SFM category as specimens showing cytologic features usually found in malignant lesions but insufficient either in quality or quantity for a definitive diagnosis of malignancy. Hou et al[6] has shown that the ROM for SFM was significantly higher than that for AUS $(P<0.01)$, which supports the separate diagnostic categories of these two independent groups, while a key question to ask is whether SFM category based on heterogeneous cytological features (quality or quantity) carries the same ROM and deserve the same clinical management.

Up to now, only a few publications supported the use of a particular terminology for serous effusion cytology[6-15]. In this study, we retrospectively applied TIS recommendations for reporting the cytological diagnosis of serous effusions. After samples reclassification, the risk of malignancy (ROM) for each TIS category was calculated, and the performance evaluation was carried out between different sample preparations (conventional smears, liquid-based preparations and cell-blocks). For all we know, this is the first publication looking into the method of preparation employed of a retrospective cohort of serous effusions based on the TIS. Besides, we sought to review and subclassify the SFM category into 2 groups (IVa and IVb) by the cytological features (quality and quantity) to calculate the ROM of each subgroup and to evaluate the necessity of having a subclassification for the SFM category.

\section{Materials And Methods}

\section{Data collection}

The study was approved by the Ethics Committee of the Peking University Cancer Hospital. The inclusion criteria were cytopathological samples of serous effusions (pleural, peritoneal and pericardial effusions) from Peking University Cancer Hospital from January 2018 to September 2021. Due to the artificial/iatrogenic origin, peritoneal washings were excluded in current study. Data was collected from pathology databases and electronic medical records, including patient demographics, clinical presentation, cytology and histology reports, ancillary studies and patient management. The cases were reclassified based on the microscopic description of the sample, the final diagnosis and codification of the cytology report. If the information contained in the report was considered insufficient, the original slides were reviewed by two experienced cytopathologists (Yanli Zhu and Wenhao Ren) and classified in the most suitable TIS category. All cases were reclassified blindly by the two cytopathologists according to the criteria defined in the TIS. When two cytopathologists did not agree on the reclassification of a particular case, they reached a consensus after discussion.

Preparation of the specimens 
Specimens were received fresh and were either entirely submitted for centrifugation or a representative $100 \mathrm{ml}$ sample was processed. During processing, the samples were divided into 2 tubes and centrifuged at 2500 revolutions per minute for 10 minutes. In addition, the supernatant was decanted. One of the tubes was prepared as conventional smears stained with hematoxylin-eosin and as liquid-based cytology samples using the ThinPrep method stained with Papanicolaou stain. A cell pellet was obtained from the other tube and the material was fixed in formalin, processed as a cell block, and stained with hematoxylin-eosin. A cell block is routinely prepared for all samples unless there is inadequate material.

\section{Criteria used for each TIS category}

Recategorization was performed using the TIS classification, and cases were allocated to one of the five proposed categories. The following criteria were used for reclassification:

I. Non-diagnostic (ND): Specimens with insufficient cellular elements for cytologic interpretation. Generally, it would be reasonable to consider a specimen ND due to scant cellularity or excess degeneration, improper preservation and obscuring blood in the serous effusion specimens.

II. Negative for malignancy (NFM): Specimens with cellular changes that completely lack evidence of mesothelial or non-mesothelial malignancy. The morphology of the cells, including mesothelial cells, macrophages, lymphocytes, and polymorphs, were benign irrespective of the clinical history and imaging studies.

III. Atypia of undetermined significance (AUS): Specimens that lack quantitative or qualitative cytologic features to be confidently diagnosed as either benign or malignant and that exhibit sufficiently clear morphologic features to exclude the possibility of classifying them as ND. The atypical morphologic features expressed will more closely approximate benign, reactive, or degenerative features than malignant features.

IV. Suspicious for malignancy (SFM): Specimens showing cytologic features usually found in malignant lesions but insufficient either in quality or quantity for a definitive diagnosis of malignancy. The diagnosis of IVa was made when there were rare cells displaying moderate-to-severely atypical features that were qualitatively insufficient to confidently exclude malignancy. And the diagnosis of IVb was made when there were rare cells displaying severely atypical features that were suspicious for malignancy but quantitatively insufficient for ancillary studies.

V. Malignancy (MAL): Specimens showing cytomorphologic features that, either alone or combined with the results from ancillary studies, are diagnostic of a primary (mesothelioma) or secondary (metastatic) malignancy. This category, irrespective of the history, shows medium to high cellularity with malignant cells in clusters and scattered singly on the cytosmears.

\section{Statistical analysis}

Statistical analysis was performed using IBM SPSS Statistics (Version 20.0; IBM Corp., New York, USA). The variables were mainly categorical, and the test used was the Chi-square test. A $P$-value less than 0.05 was considered significant. The gold standard for true diagnosis was based on a histological diagnoses or clinical diagnoses. The histological diagnoses were the biopsy or postoperative pathological results of the pleura, peritoneum, or pericardium corresponding to the effusion, and the clinical diagnoses were made in combination with clinical manifestations, laboratory results and medical imaging examination results. All histological and clinical diagnoses were performed independently and blindly by two physicians. For cases with inconsistent results, the same diagnosis was made after discussion by two physicians.

Performance analysis included the calculation of sensitivity, specificity, positive predictive value (PPV), negative predictive value (NPV) and diagnostic accuracy for different samples (pleural, peritoneal and pericardial effusion cytology samples) and preparation methods (conventional smears, liquidbased preparations and cell-blocks). During performance analysis, the results were calculated separately according to MAL as positives, MAL and SFM as positives, MAL, SFM and AUS as positives. ND cytology samples were excluded from performance analysis, cases which conducted necessary laboratory test and medical imaging examination, but still unable to determine whether the serous cavity was invaded were excluded.

\section{Results}

\section{Patient demography and clinicopathologic data}

Between January 2018 and September 2021, a total of 3633 serous effusions were diagnosed at our institution, including 2366 (65.1\%) pleural effusions, 1150 (31.7\%) peritoneal effusions and 117 (3.2\%) pericardial effusions. The mean age and male to female ratio were 58.7 (ranged $9-93$ years) and 0.96 , respectively. The volume of serous effusions ranged from 20 to $1000 \mathrm{ml}$ (mean: $265 \mathrm{ml}$ ). Cell-blocks were prepared in 2882 cases. Of all specimens, 17 (0.5\%) were diagnosed as ND, 1100 (30.3\%) as NFM, 101 (2.8\%) as AUS, 677 (18.6\%) as SFM, and 1738 (47.8\%) as MAL. The patient demographics and specimen characteristics are shown in Table 1.

\section{Risk of malignancy}

Integrating the cytological diagnoses with other ancillary diagnostic tests performed, histological diagnoses and clinical data, 45 cases were excluded for their follow-up diagnoses were inconclusive or inadequate. The follow-up data were used for the calculation of the ROM for each category in 3588 cases. Table 2 depicts the calculated ROM for each category, and Table 3 provides the subclassification of the indeterminate categories of AUS and SFM and the corresponding ROMs. 
The ROM and performance analysis in pleural, peritoneal and pericardial effusion specimens

\section{Pleural effusions}

In total, 2326 cases of pleural effusions were reclassified in accordance with criteria set by the TIS: 10 (0.4\%) ND, 691 (29.7\%) NFM, 73 (3.1\%) AUS, 442 (19.0\%) SFM, 1110 (47.7\%) MAL. When considering only MAL as positive, false negatives were found in 681 cases while there were no false positive cases. When considering MAL and SFM as positives, false negatives were found in 242 cases while three false positive cases were found(Figure 1), possible reasons for those false positives were outlined in the figure1 description. The ROM for ND was 40\%(4/10), 29.8\%(206/691) for NFM, 49.3\%(36/73) for AUS, 99.3\%(439/442) for SFM and 100\%(1110/1110) for MAL. Considering only MAL as positive cases, sensitivity was $62.0 \%$, specificity was $100 \%$, PPV was $100 \%$, NPV was $43.5 \%$ and diagnostic accuracy was $70.6 \%$. Considering MAL and SFM as positive, sensitivity was $86.5 \%$, specificity was $99.4 \%$, PPV was $99.8 \%$, NPV was $68.3 \%$ and diagnostic accuracy was $89.4 \%$. Considering MAL, SFM and AUS as positive, sensitivity was $88.5 \%$, specificity was $92.4 \%$, PPV was $97.5 \%$, NPV was $70.2 \%$ and diagnostic accuracy was $89.4 \%$.

\section{Peritoneal effusions}

In total, 1145 peritoneal effusion samples were reclassified by the TIS: 2 (0.2\%) ND, 327 (28.6\%) NFM, 23 (2.0\%) AUS, 222 (19.4\%) SFM and 571 (49.9\%) MAL. Considering only MAL as positive, false negatives were found in 325 cases while there were no false positive cases. When considering MAL and SFM as positives, false negatives were found in 104 cases while one false positive case was found(Figure 2), possible reason for the false positive was outlined in the figure2 description. The ROM was 0\%(0/2) for ND, 27.5\%(90/327) for NFM, 60.9\%(14/23) for AUS, 99.5\%(221/222) for SFM and $100 \%(571 / 571)$ for MAL. Considering only MAL as positive cases, sensitivity was $63.7 \%$, specificity was $100 \%$, PPV was $100 \%$, NPV was 43.2\% and diagnostic accuracy was $71.6 \%$. Considering MAL and SFM as positive, sensitivity was $88.4 \%$, specificity was $99.6 \%$, PPV was $99.9 \%$, NPV was $70.3 \%$ and diagnostic accuracy was $90.8 \%$. Considering MAL, SFM and AUS as positive, sensitivity was $90.0 \%$, specificity was $96.0 \%$, PPV was $98.8 \%$, NPV was $72.5 \%$ and diagnostic accuracy was $91.3 \%$.

\section{Pericardial effusions}

117 cases were reclassified by the TIS: 1 (0.9\%) ND, 46 (39.3\%) NFM, 13 (11.1\%) SFM and 57 (48.7\%) MAL, there were no cases for AUS. When considering MAL as positive and MAL and SFM as positives, there were both no false positive cases, and false negatives were found in 21 cases and 8 cases, respectively. The ROM for ND was 100\%(1/1), 17.4\%(8/46) for NFM, 100\%(13/13) for SFM and 100\%(57/57) for MAL. Considering only MAL as positive cases, sensitivity was $73.1 \%$, specificity was $100 \%$, PPV was $100 \%$, NPV was $64.4 \%$ and diagnostic accuracy was $81.9 \%$. Considering MAL and SFM as positive, sensitivity was $89.7 \%$, specificity was $100 \%$, PPV was $100 \%$, NPV was $82.6 \%$ and diagnostic accuracy was $93.1 \%$. Considering MAL, SFM and AUS as positive, sensitivity was $89.7 \%$, specificity was $100 \%$, PPV was $100 \%$, NPV was $82.6 \%$ and diagnostic accuracy was $93.1 \%$. Table 4 presents the results of the performance analysis among different serous effusions in current study and previous publications.

\section{The performance analysis among different sample preparations}

The diagnoses of 1288 cases, containing conventional smear, liquid-based preparation and cell block at the same time, according each preparation method were recorded in our pathology database. The data were collected and the performance evaluation among different preparation methods were analyzed. In our analysis, the sensitivity, NPV and diagnostic accuracy of liquid-based preparations were all superior to conventional smears and cellblocks in detecting abnormalities (Figure 3 and Figure 4). Besides, we found applying two methods at the same time preceded to any single method and using three methods at the same time had the highest sensitivity, NPV and diagnostic accuracy, while there was little difference between two diverse methods of preparation (Figure 3 and Figure 4).

\section{Discussion}

Serous effusion cytology is a minimally invasive and cost-effective diagnostic method used to investigate etiologies of body cavity effusions and can guide clinical decision-making. And to the best of our knowledge, this is the largest series to date of such analyses in serous effusions. We evaluated our application of the recently proposed TIS on reporting serous effusion cytopathology. A total of 3633 patients were included, among which 17 (0.5\%), 1100 (30.3\%), 101 (2.8\%), 677 (18.6\%) and 1738 (47.8\%) in patients were classified into ND, NFM, AUS, SFM and MAL groups, respectively.

The malignancy rate (47.8\%) detected in our cohort was higher than that reported in the literature, which ranges between 4\% and $22.4 \%[6-9,11-13,15]$ but was similar to other reports in literature from oncological centers[6,10,14]. The percentage of our SFM cases was also slightly higher than other reported studies (range: SFM, 1.3\%-6.3\%)[6-15]. These results are characteristic of a cancer center, where neoplastic conditions are the main cause of cytological examination of body fluids, and if one case is diagnosed as SFM, the clinicians will consider it as positive, taking the history into account, hence, the case is failed to be identified malignancy by auxiliary examination. Moreover, our study included both outpatient and hospitalized patients, and the outpatients often failed to be identified malignancy by auxiliary examination. Besides, we didn't use a specific number of cells to determine whether a sample is suspicious or positive for malignancy. In patients with a clear history of disseminated malignancy, although there is no immunochemistry support, few highly atypical cells might be sufficient to diagnose SFM. In other clinical situation, the same number of cells might not be enough for a diagnosis of SFM and can only be diagnosed as AUS. If we follow the principles of clinical management, $96.6 \%$ of our serous 
effusions received a directed diagnosis, including NFM, MAL and SFM, and only a small proportion of cases were diagnosed as ND and AUS (0.5\%, $2.7 \%)$.

Our non-diagnostic rate was $0.5 \%$. This is in line with other reports in literature, which present nondiagnostic rates of $0 \%$ to $5.6 \%$ in serous effusion[615]. The cases classified as ND in this series were all due to scant cellularity or excess degeneration, improper preservation and obscuring blood in the serous effusion specimens. Therefore, the whole blood ones should be anticoagulated timely before sample preparation.

The minimal threshold of adequacy for fluid interpretation is still contentious and has not been described clearly in the TIS. Some earlier studies have suggested a minimum of 50-75 ml[16-18], but the evidence is limited. Recently, Gokozan et al performed a root cause analysis of the diagnoses of atypia or suspicious for malignancy and showed $50 \mathrm{~mL}$ and below were considered low volume samples, and were included as a root cause for indeterminate diagnoses[8]. In the present study, the 17 non-diagnostic cases all had a specimen volume greater than or equal to $50 \mathrm{ml}$ and many of our malignant cases had a very low volume submitted to our laboratory. Therefore, the volume threshold of adequacy should be regarded as a recommendation, with the final decision left to each practice.

In our cohort, the ROM values of serous effusions in ND, NFM, AUS, SFM and MAL groups were $38.5 \%, 28.6 \%, 52.1 \%, 99.4 \%$ and $100 \%$, respectively. It is worth noting that high ROMs can be seen in our study for SFM. One possible reason for this result could be due to the fact that our data comes from a large cancer center, and the majority of the serous effusions often originate from tumors. For the cases with high clinical suspicion, even though cytology was negative, patients usually underwent medical imaging examination and clinicians tended to pursue pleural biopsies, which were used in the present study as the gold standard for the calculation of ROM, providing a potential selection bias. Our high ROM for SFM support the viewpoint that most clinicians will manage patients with SFM effusions similar to the manner in which they manage those with a malignant diagnosis. Besides, in our study, the ROMs for the non-diagnostic and negative categories were also high, which may also be attributed to the nature of the patient population in our cancer center, many of whom were referred to our hospital with an established malignant diagnosis and frequently at an advanced stage of disease.

The TIS describes two indeterminate categories: AUS and SFM, created to encompass all the fluid that could not be placed under the NFM or MAL categories. In our study, the ROM for SFM was significantly higher than that for AUS $(P<0.001)$, thus providing support for retaining the two indeterminate categories as independent ones. While the difference between the ROMs for the IVa and IVb was insignificant with the $P$-value of 0.124 , which indicate that although different situations exist that can be diagnosed in the SFM category, there is no need to reclassify for the SFM category. From the Table 2, it is worth noting the wide range of ROM calculated for each diagnostic category and that is likely attributable to the variation of reporting among different institutions. The standardized reporting would also provide a meaningful language that clinicians can uniformly understand and utilize in their patient management. More research is needed to convey their ROM of each category to the corresponding clinical colleagues to optimize patient care.

Our performance analysis was in agreement with previous publications[10, 11, 13, 14, 19] (Table 4). By comparing the performance evaluation of different groups (considering cytological diagnosis of MAL+SFM+AUS as positives, considering cytological diagnosis of MAL+SFM as positives, and considering cytological diagnosis of MAL as positive), it is best to consider the categories of MAL and SFM as positives, while excluding AUS, to be beneficial to clinical management. This is supported by our data (Table 4) and agree with the perspective of TIS[5]. What's more, it is worth mentioning that when considering the categories of MAL and SFM as positives, there were only 4 false positive cases, and the 4 cases were all diagnosed as IVa, and the total specificity and the PPV can be as high as $99.5 \%$ and $99.8 \%$, respectively.

Compared with conventional smears, liquid-based preparations require less skills, and more importantly, allow for the application of ancillary examinations[20]. Moreover, liquid-based preparations permit for more evenly distribution of cells over the slide area, reduction of obscuring background elements, and better preservation of nuclear detail and cytoplasm. Several studies have shown slides prepared with liquid-based preparations have a lower non-diagnostic incidence and higher accuracy than conventional smears[21-23]. In our study, the sensitivity, NPV and diagnostic accuracy of liquid-based preparations were all superior to conventional smears and cell-blocks in detecting abnormalities. Besides, by comparing the performance evaluation among different methods of preparation, we found applying two methods at the same time preceded to any single method and using three methods simultaneously had the highest sensitivity, NPV and diagnostic accuracy, while there was little difference between two diverse methods of preparation. Therefore, in terms of the selection of preparation methods, it is best to use three different methods simultaneously for serous effusion cytologic examination.

There are 2 main limitations in our study. The first is attributed to the nature of the patient population in our cancer center, leading to high rate of MAL and SFM, as well as high ROM for each category. Another limitation of our study is this is a unicentric and retrospective study and the cytologic diagnoses were signed out by two cytopathologists, who although reclassifying cases according to the TIS criteria, having their own personal deviations.

\section{Conclusions}

In conclusion, this study supports the idea of retaining the two indeterminate categories (AUS and SFM) as independent ones, and there is no need to reclassify for the SFM category. By comparing the performance evaluation of different groups, it is best to consider the categories of MAL and SFM as positives, while excluding AUS. $96.6 \%$ of our serous effusions received a directed diagnosis, including NFM, MAL and SFM, and only a small 
proportion of cases were diagnosed as ND and AUS (0.5\%, 2.7\%), demonstrating the TIS is a user-friendly reporting system. The sensitivity, NPV and diagnostic accuracy of liquid-based preparations were all superior to conventional smears and cell-blocks in detecting abnormalities. And it is best to use three different preparation methods simultaneously for serous effusion cytologic examination.

\section{Abbreviations}

AF: Ascitic fluid; AUS: Atypia of undetermined significance; MAL: Malignancy; ND: Non-diagnostic; NFM: Negative for malignancy; NPV: Negative predictive value; PeriF: Pericardial fluid; PF: Pleural fluid; PPV: Positive predictive value; ROM: Risk of malignancy; SFM: Suspicious of malignancy; TIS: The International System for Reporting Serous Fluid Cytopathology.

\section{Declarations}

\section{Ethics approval and consent to participate}

All patient samples and clinical data using were approved by the Ethics committee of the Peking University Cancer Hospital and the exemption from informed consent was approved as well.

\section{Consent for publication}

Not applicable.

\section{Availability of data and materials}

The datasets used and/or analyzed during the current study are available from the corresponding author on reasonable request.

\section{Competing interests}

The authors declare that they have no competing interest.

\section{Funding}

Not applicable.

\section{Author contributions}

Yan-li Zhu and Wen-hao Ren contributed equally as first author. Yan-li Zhu conceived the project idea. Qian wang, Hai-zhu Jin and Yi-yi Guo performed smear/section preparation. Dong-mei Lin and Yan-li Zhu were responsible for cytological and histological analysis. All authors interpreted and discussed the results. Yan-li Zhu, Wen-hao Ren and Dong-mei Lin wrote the manuscript. All authors have read and approved the final version.

\section{Acknowledgements}

Not applicable.

\section{Author details}

Key laboratory of Carcinogenesis and Translational Research (Ministry of Education), Department of Pathology, Peking University Cancer Hospital and Institute, Beijing 100142, China.

\section{References}

1. Ebata T, Okuma Y, Nakahara $Y$, Yomota M, Takagi $Y$, Hosomi $Y$, et al. Retrospective analysis of unknown primary cancers with malignant pleural effusion at initial diagnosis. Thorac Cancer. 2016;7(1):39-43.

2. Zamboni MM, da Silva CT, Jr., Baretta R, Cunha ET, Cardoso GP. Important prognostic factors for survival in patients with malignant pleural effusion. BMC Pulm Med. 2015;15:29.

3. Sangisetty SL, Miner TJ. Malignant ascites: A review of prognostic factors, pathophysiology and therapeutic measures. World J Gastrointest Surg. 2012;4(4):87-95.

4. Crothers BA, Chandra A. Proceedings of the American Society of Cytopathology Companion Session at the 2019 United States and Canadian Academy of Pathology Meeting Part 1: towards an International System for Reporting Serous Fluid Cytopathology. J Am Soc Cytopathol. 2019;8(6):362-8.

5. Chandra A, Crothers B, Kurtycz D, Schmitt F. Announcement: The International System for Reporting Serous Fluid Cytopathology. Acta Cytologica. 2019;63:1-3. 
6. Hou T, Landon G, Stewart J, Roy-Chowdhuri S. The value of a tiered cytology diagnostic reporting system in assessing the risk of malignancy in indeterminate serous effusions. Cancer Cytopathol. 2021;129(1):75-82.

7. Savari O, Jassim S, Ferrer H, Ganocy SJ, Ganesan S. Role of CD68 immunohistochemistry in categorizing benign nonmesothelial cell population and refining "atypical" category in serous fluid cytology. Diagn Cytopathol. 2020;48(12):1199-204.

8. Gokozan HN, Harbhajanka A, Lyden S, Michael CW. Root cause analysis of indeterminate diagnoses in serous fluids cytopathology. Diagn Cytopathol. 2021;49(5):633-9.

9. Rodriguez EF, Jones R, Gabrielson M, Santos D, Pastorello RG, Maleki Z. Application of the International System for Reporting Serous Fluid Cytopathology (ISRSFC) on Reporting Pericardial Effusion Cytology. Acta Cytol. 2020;64(5):477-85.

10. Lobo C, Costa J, Petronilho S, Monteiro P, Leça L, Schmitt F. Cytohistological correlation in serous effusions using the newly proposed International System for Reporting Serous Fluid Cytopathology: Experience of an oncological center. Diagn Cytopathol. 2021;49(5):596-605.

11. Xu Y, Hu AY, Wang SM, Wang Q, Pan YC, Zhang SH. A retrospective analysis of pleural effusion specimens based on the newly proposed International System for Reporting Serous Fluid Cytopathology. Diagn Cytopathol. 2021;49(9):997-1007.

12. Jha S, Sethy M, Adhya AK. Application of the International System for Reporting Serous Fluid Cytopathology in routine reporting of pleural effusion and assessment of the risk of malignancy. Diagn Cytopathol. 2021;49(10):1089-98.

13. Pinto D, Cruz E, Branco D, Linares C, Carvalho C, Silva A, et al. Cytohistological Correlation in Pleural Effusions Based on the International System for Reporting Serous Fluid Cytopathology. Diagnostics (Basel). 2021;11(6).

14. Valerio E, Nunes W, Cardoso J, Santos A, Bovolim G, Domingos T, et al. A 2-year retrospective study on pleural effusions: A cancer centre experience. Cytopathology. 2019;30(6):607-13.

15. Farahani SJ, Baloch Z. Are we ready to develop a tiered scheme for the effusion cytology? A comprehensive review and analysis of the literature. Diagn Cytopathol. 2019;47(11):1145-59.

16. Abouzgheib W, Bartter T, Dagher H, Pratter M, Klump W. A prospective study of the volume of pleural fluid required for accurate diagnosis of malignant pleural effusion. Chest. 2009;135(4):999-1001.

17. Thomas SC, Davidson LR, McKean ME. An investigation of adequate volume for the diagnosis of malignancy in pleural fluids. Cytopathology. 2011;22(3):179-83.

18. Rooper LM, Ali SZ, Olson MT. A minimum fluid volume of $75 \mathrm{~mL}$ is needed to ensure adequacy in a pleural effusion: a retrospective analysis of 2540 cases. Cancer Cytopathol. 2014;122(9):657-65.

19. Rossi ED, Bizzarro T, Schmitt F, Longatto-Filho A. The role of liquid-based cytology and ancillary techniques in pleural and pericardic effusions: an institutional experience. Cancer Cytopathol. 2015;123(4):258-66.

20. Cox JT. Liquid-based cytology: evaluation of effectiveness, cost-effectiveness, and application to present practice. J Natl Compr Canc Netw. 2004;2(6):597-611.

21. Ronco G, Cuzick J, Pierotti P, Cariaggi MP, Dalla Palma P, Naldoni C, et al. Accuracy of liquid based versus conventional cytology: overall results of new technologies for cervical cancer screening: randomised controlled trial. Bmj. 2007;335(7609):28.

22. Yeon MH, Jeong HS, Lee HS, Jang JS, Lee S, Yoon SM, et al. Comparison of liquid-based cytology (CellPrepPlus) and conventional smears in pancreaticobiliary disease. Korean J Intern Med. 2018;33(5):883-92.

23. Halford JA, Batty T, Boost T, Duhig J, Hall J, Lee C, et al. Comparison of the sensitivity of conventional cytology and the ThinPrep Imaging System for 1,083 biopsy confirmed high-grade squamous lesions. Diagn Cytopathol. 2010;38(5):318-26.

\section{Tables}

Table 1. Patient demographics and specimen characteristics of 3633 serous effusions based on each TIS category. 


\begin{tabular}{|c|c|c|c|c|c|c|}
\hline Diagnostic category & ND & NFM & AUS & SFM & MAL & Total \\
\hline Number of patients(n, percentage) & $17(0.5 \%)$ & $1100(30.3 \%)$ & $101(2.8 \%)$ & $677(18.6 \%)$ & $1738(47.8 \%)$ & 3633 \\
\hline Outpatient/inpatient & $5 / 12$ & 203/897 & $24 / 77$ & $158 / 519$ & $436 / 1302$ & $826 / 2807$ \\
\hline Gender(number of men/women) & $11 / 6$ & $656 / 444$ & $66 / 35$ & $341 / 336$ & $703 / 1035$ & $1777 / 1856$ \\
\hline Average age(year, ranges) & $53(40-65)$ & $59(17-93)$ & $61(9-90)$ & $59(17-93)$ & $59(14-92)$ & $58.7(9-93)$ \\
\hline \multicolumn{7}{|l|}{ Serous effusion source(n) } \\
\hline Pleural & 14 & 723 & 77 & 442 & 1110 & 2366 \\
\hline Peritoneal & 2 & 331 & 24 & 222 & 571 & 1150 \\
\hline Pericardial & 1 & 46 & 0 & 13 & 57 & 117 \\
\hline
\end{tabular}

Abbreviations: ND, non-diagnostic; NFM, negative for malignancy; AUS, atypia of undetermined significance; SFM, suspicious of malignancy; MAL, malignancy.

Table 2. The risk of malignancy in current study and comparison with a few previous publications.

\begin{tabular}{|c|c|c|c|c|c|c|c|c|}
\hline \multirow[t]{2}{*}{ Author } & \multirow{2}{*}{$\begin{array}{l}\text { Specimen } \\
\text { type }\end{array}$} & \multirow[t]{2}{*}{ Year } & \multirow[t]{2}{*}{ Total cases } & \multicolumn{5}{|c|}{ Risk of malignancy(ROM) } \\
\hline & & & & ND & NFM & AUS & SFM & MAL \\
\hline $\begin{array}{l}\text { Sahar } \\
\text { J. Fet } \\
\text { al }\end{array}$ & $\mathrm{PF}+\mathrm{AF}+\mathrm{PeriF}$ & 2019 & 34941 & $17.4 \%$ & $20.7 \%$ & $65.9 \%$ & $81.8 \%$ & $98.9 \%$ \\
\hline $\begin{array}{l}\text { Ediel } V \\
\text { et al }{ }^{14}\end{array}$ & $\mathrm{PF}$ & 2019 & 519 & $50 \%$ & $44 \%$ & $50 \%$ & $83.3 \%$ & $96.2 \%$ \\
\hline $\begin{array}{l}\text { Cláudia } \\
L \text { et } \\
a l^{10}\end{array}$ & PF/AF/ PeriF & 2020 & $1496 / 763 / 64$ & $57.1 \% / 100 \% /-$ & $23.9 \% / 26.3 \% / 0 \%$ & $50 \% / 62.5 \% / 0 \%$ & $76.2 \% / 91.7 \% /-$ & $100 \% / 100 \% / 100 \%$ \\
\hline $\begin{array}{l}\text { YiX et } \\
a l^{11}\end{array}$ & $\mathrm{PF}$ & 2021 & 2454 & $26.7 \%$ & $12 \%$ & $62.3 \%$ & $77.8 \%$ & $100 \%$ \\
\hline $\begin{array}{l}\text { Shilpy } \\
\text { Jet } \\
a l^{12}\end{array}$ & $\mathrm{PF}$ & 2021 & 939 & $87.5 \%$ & $51.6 \%$ & $88.2 \%$ & $87.5 \%$ & $100 \%$ \\
\hline $\begin{array}{l}\text { Daniel } \\
\text { Pet } \\
\text { al }\end{array}$ & $\mathrm{PF}$ & 2021 & 350 & $40 \%$ & $20.16 \%$ & $42.86 \%$ & $78.57 \%$ & $100 \%$ \\
\hline $\begin{array}{l}\text { Tieying } \\
\text { Het al }\end{array}$ & $\mathrm{PF}+\mathrm{AF}+\mathrm{PeriF}$ & 2021 & 2405 & - & - & $39 \%$ & $64 \%$ & - \\
\hline $\begin{array}{l}\text { Current } \\
\text { study }\end{array}$ & $\mathrm{PF}+\mathrm{AF}+\mathrm{PeriF}$ & 2021 & 3588 & $38.5 \%(5 / 13)$ & $\begin{array}{l}28.6 \% \\
(304 / 1064)\end{array}$ & $52.1 \%(50 / 96)$ & $\begin{array}{l}99.4 \% \\
(673 / 677)\end{array}$ & $100 \%(1738 / 1738)$ \\
\hline & $\mathrm{PF}$ & & 2326 & $40 \%(4 / 10)$ & $29.8 \%(206 / 691)$ & $49.3 \%(36 / 73)$ & $\begin{array}{l}99.3 \% \\
(439 / 442)\end{array}$ & $100 \%(1110 / 1110)$ \\
\hline & $\mathrm{AF}$ & & 1145 & $0 \%(0 / 2)$ & $27.5 \%(90 / 327)$ & $60.9 \%(14 / 23)$ & $\begin{array}{l}99.5 \% \\
(221 / 222)\end{array}$ & $100 \%(571 / 571)$ \\
\hline & PeriF & & 117 & $100 \%(1 / 1)$ & $17.4 \%(8 / 46)$ & $0 \%(0 / 0)$ & $100 \%(13 / 13)$ & $100 \%(57 / 57)$ \\
\hline
\end{tabular}

Abbreviations: ND, non-diagnostic; NFM, negative for malignancy; AUS, atypia of undetermined significance; SFM, suspicious of malignancy; MAL, malignancy; PF, pleural fluid; AF, ascitic fluid; PeriF, pericardial fluid.

Table 3. Subclassification of the indeterminate categories of AUS and SFM and the corresponding risk of malignancy(ROM). 


\begin{tabular}{lllll} 
Subclassification of AUS/SFM & No.(\%) & \multicolumn{2}{l}{ Surgical pathology/clinical diagnosis } & ROM \\
\cline { 3 - 5 } & & Benign & Malignant & \\
\hline AUS & 96 & 46 & 50 & $52.1 \%$ \\
\hline SFM & 677 & 4 & 673 & $99.4 \%$ \\
\hline SFM-A & 333 & 4 & 329 & $98.8 \%$ \\
\hline SFM-B & 344 & 0 & 344 & $100 \%$ \\
\hline Total & 773 & 50 & 723 & $93.5 \%$
\end{tabular}

Abbreviations: AUS, atypia of undetermined significance; SFM: suspicious of malignancy;

Note: SFM-A: There were rare cells displaying moderate-to-severely atypical features that were qualitatively insufficient to confidently exclude malignancy; SFM-B: There were rare cells displaying severely atypical features that were suspicious for malignancy but quantitatively insufficient for ancillary studies.

Table 4. The performance analysis among different serous effusions in current study and previous publications. 


\begin{tabular}{|c|c|c|c|c|c|}
\hline & & & \multicolumn{3}{|l|}{ Positive standard } \\
\hline & & & MAL+SFM+AUS & MAL+SFM & MAL \\
\hline \multirow[t]{10}{*}{ Pleural effusions } & Current study & Sensitivity & $88.5 \%$ & $86.5 \%$ & $62.0 \%$ \\
\hline & & Specificity & $92.4 \%$ & $99.4 \%$ & $100.0 \%$ \\
\hline & & PPV & $97.5 \%$ & $99.8 \%$ & $100.0 \%$ \\
\hline & & NPV & $70.2 \%$ & $68.3 \%$ & $43.5 \%$ \\
\hline & & Diagnostic accuracy & $89.4 \%$ & $89.4 \%$ & $70.6 \%$ \\
\hline & Previous publications & Sensitivity & - & $60.3 \%-99.7 \% 11,13$ & $61.6 \%-87 \% 10-11,14,19$ \\
\hline & & Specificity & - & $98.6 \%-99.4 \% 11,13$ & $93.3 \%-100 \% 10-11,14,19$ \\
\hline & & $P P V$ & - & $96.5 \%-98.3 \% 11,13$ & $96.2 \%-100 \% 10-11,14,19$ \\
\hline & & $N P V$ & - & $79.2 \%-99.9 \% 11,13$ & $56 \%-98 \% 10-11,14,19$ \\
\hline & & Diagnostic accuracy & - & $97 \% 11$ & $81.3 \%-98 \% 10-11,14,19$ \\
\hline \multirow[t]{10}{*}{ Peritoneal effusions } & Current study & Sensitivity & $90.0 \%$ & $88.4 \%$ & $63.7 \%$ \\
\hline & & Specificity & $96.0 \%$ & $99.6 \%$ & $100.0 \%$ \\
\hline & & PPV & $98.8 \%$ & $99.9 \%$ & $100.0 \%$ \\
\hline & & NPV & $72.5 \%$ & $70.3 \%$ & $43.2 \%$ \\
\hline & & Diagnostic accuracy & $91.3 \%$ & $90.8 \%$ & $71.6 \%$ \\
\hline & Previous publications & Sensitivity & - & - & $61.2 \% 10$ \\
\hline & & Specificity & - & - & $100.0 \% 10$ \\
\hline & & $P P V$ & - & - & $100.0 \% 10$ \\
\hline & & $N P V$ & - & - & $70.0 \% 10$ \\
\hline & & Diagnostic accuracy & - & - & $79.7 \% 10$ \\
\hline \multirow[t]{10}{*}{ Pericardial effusions } & Current study & Sensitivity & $89.7 \%$ & $89.7 \%$ & $73.1 \%$ \\
\hline & & Specificity & $100.0 \%$ & $100.0 \%$ & $100.0 \%$ \\
\hline & & PPV & $100.0 \%$ & $100.0 \%$ & $100.0 \%$ \\
\hline & & NPV & $82.6 \%$ & $82.6 \%$ & $64.4 \%$ \\
\hline & & Diagnostic accuracy & $93.1 \%$ & $93.1 \%$ & $81.9 \%$ \\
\hline & Previous publications & Sensitivity & - & - & $97 \%-100 \% 10,19$ \\
\hline & & Specificity & - & - & $100 \% 10,19$ \\
\hline & & $P P V$ & - & - & $100 \% 10,19$ \\
\hline & & $N P V$ & - & - & $99 \%-100 \% 10,19$ \\
\hline & & Diagnostic accuracy & - & - & $99 \%-100 \% 10,19$ \\
\hline \multirow[t]{4}{*}{ Total effusions } & Current study & Sensitivity & $89.0 \%$ & $87.2 \%$ & $62.9 \%$ \\
\hline & & Specificity & $93.8 \%$ & $99.5 \%$ & $100.0 \%$ \\
\hline & & PPV & $98.0 \%$ & $99.8 \%$ & $100.0 \%$ \\
\hline & & NPV & $71.4 \%$ & $69.5 \%$ & $44.1 \%$ \\
\hline
\end{tabular}




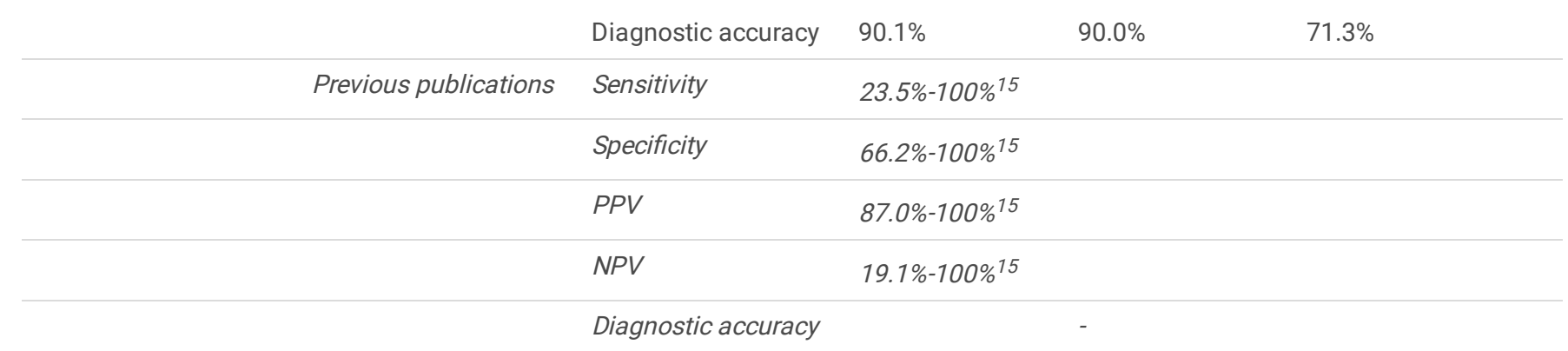

Abbreviations: AUS, atypia of undetermined significance; SFM, suspicious for malignancy; MAL, malignancy; PPV, positive predictive value; NPV, negative predictive value.

\section{Figures}

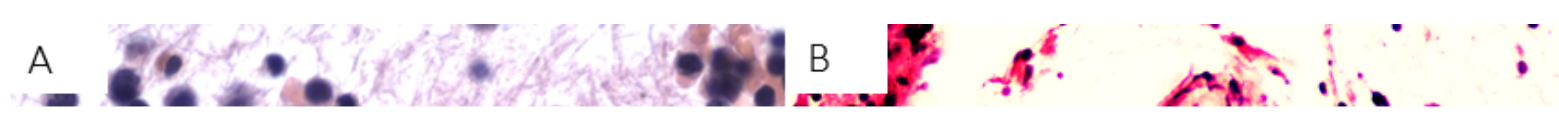

Figure 1

False positive cases in pleural effusion specimens.

Case1: The cytological diagnosis was 'not exclusion of malignancy'(A, TIS category: IVa, HE, $\times 400)$, while the histological diagnosis was granulomatous lesions $(\mathrm{B}, \mathrm{HE}, \times 200)$; We think it may be that the epithelioid cells in the granulomatous lesions (bottom right of the figure $B$ ) were mistaken for cells that'not exclusion of malignancy'.

Case2: The cytological diagnosis was 'not exclusion of lymphoma'(C, TIS category: IVa, HE, $\times 100)$ while the clinical diagnosis was 'tuberculosis'; The patient has a history of abdominal lymphoma. A few weeks after the cytological diagnosis, he was clinically diagnosed with thoracic tuberculosis and pleural fluid disappeared after anti-tuberculosis treatment. It may be that the history of lymphoma led to our wrong diagnosis. 
Case3: The cytological diagnosis was 'not exclusion of malignancy'(D, TIS category: IVa, Papanicolaou stain, $\times 400)$ while the clinical diagnosis was 'chylothorax'. The misdiagnosed cells may be reactive mesothelial cells caused by chylothorax.
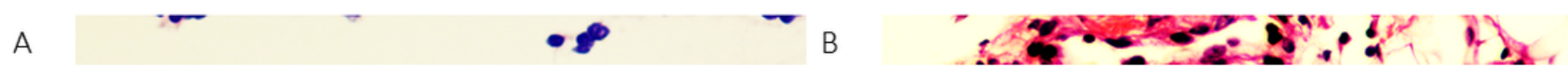

Figure 2

The false positive case in peritoneal effusion specimens. The cytological diagnosis was 'not exclusion of malignancy' (A, TIS category: Iva, HE, $\times 400)$, while the omentum biopsy result was chronic inflammation $(B, H E, \times 200)$. We think it may be that the fibroblasts (bottom right of Figure $B)$ were mistaken for cells that'not exclusion of malignancy.

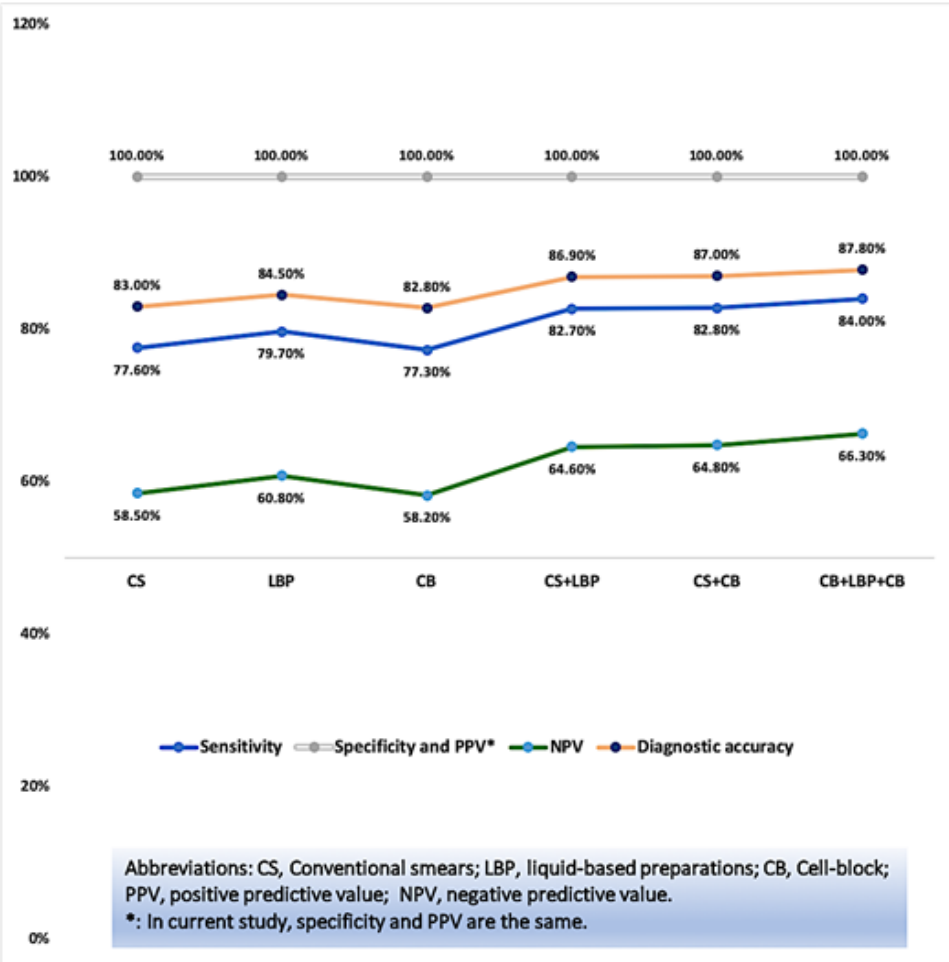

Figure 3

The performance evaluation among different sample preparations in 1288 cases when considering malignancy, suspicious of malignancy and atypia of undetermined significance as positives. 


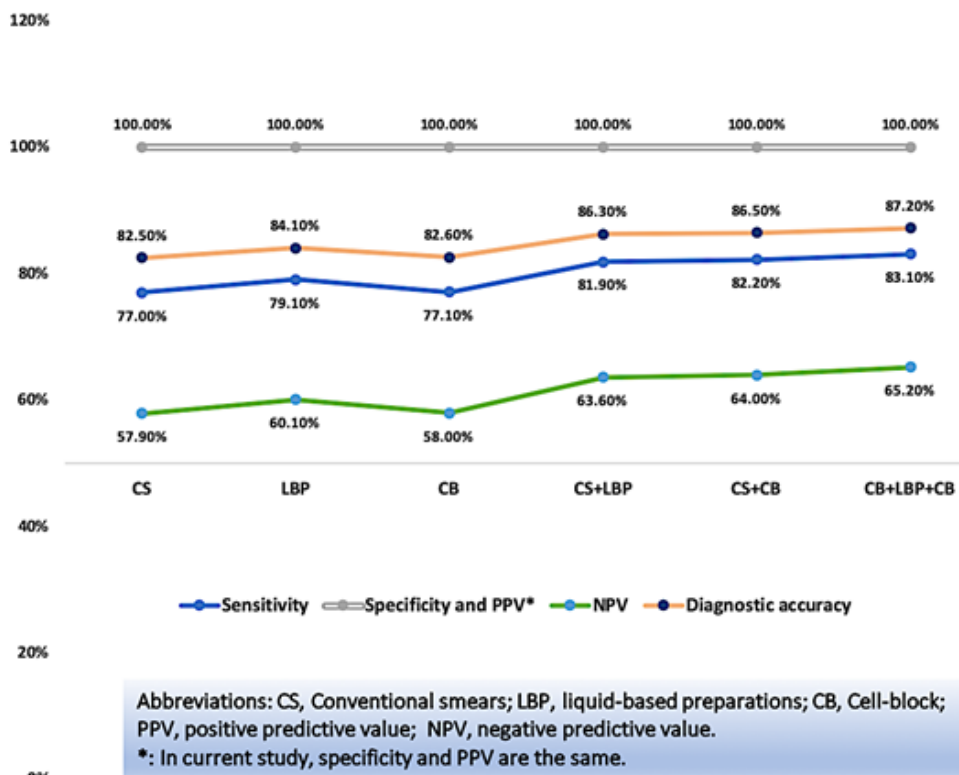

ox

\section{Figure 4}

The the performance evaluation among different sample preparations in 1288 cases when considering malignancy and suspicious of malignancy as positives. 